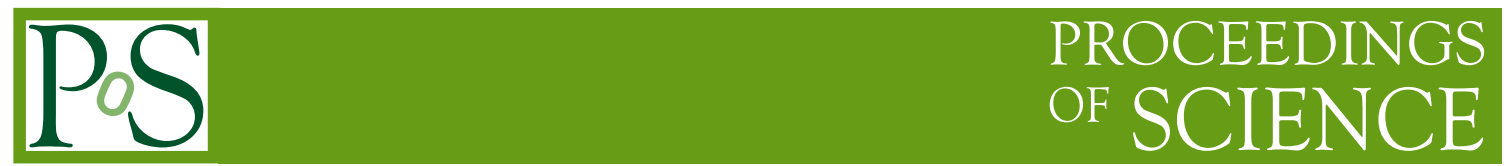

\title{
QCD at finite chemical potential with $N_{t}=8$
}

\section{Saumen Datta, Rajiv V. Gavai, Sourendu Gupta}

Department of Theoretical Physics, Tata Institute of Fundamental Research, Homi Bhabha Road, Mumbai 400005, India.

E-mail:

saumen@theory.tifr.res.in, gavai@tifr.res.in, sgupta@theory.tifr.res.in

Results from our simulations of QCD with two light dynamical staggered flavours of mass $m / T_{c}=0.1$ are presented. Employing our earlier proposed Taylor series method for the baryonic susceptibility, we estimate the radius of convergence by using terms up to the eighth order susceptibility, $\chi_{8}$. Comparing with earlier results on coarser lattices, we find a very good agreement between the $N_{t}=8$ and 6 lattices for its location in the $\left(\mu_{B} / T, T / T_{c}\right)$ plane, suggesting any cut-off effects to be encouragingly small.

31st International Symposium on Lattice Field Theory - LATTICE 2013

July 29 - August 3, 2013

Mainz, Germany 


\section{Introduction}

A major focus of many experimental programs in heavy-ion physics is the search for the critical point. A successful search would yield the critical values of the temperature, $T$, and baryon chemical potential, $\mu_{B}$. Such experimental programs include the Relativistic Heavy Ion Collider (RHIC) of BNL, New York or the upcoming FAIR facility at GSI, Darmstadt and NICA in Russia. Many effective models for QCD, including the Nambu-Jona-Lasinio model and its extensions, suggest the existence of a second order phase transition at finite chemical potential [1].

One might expect that the most reliable way to locate the critical point, if it exists, would be through lattice computations. This obvious tool, when applied at non-zero $\mu_{B}$, is constrained by formidable difficulties in addition to those encountered at $\mu_{B}=0$. Effective models suggest that having two light flavours of quarks could be crucial for the existance of the critical point. The limit of the strange quark becoming as light as the up and down quarks, in such models may not yield a critical point in the $T-\mu_{B}$ plane. Mostly simulations so far have employed staggered quarks. Due to the importance of these results to the experimental programs mentioned before, it will eventually be necessary to employ fermions with exact flavour and chiral symmetry on the lattice at arbitrary $\mu_{B}$. Recently, a lattice action with nonzero $\mu$ and the same chiral symmetries as continuum QCD has been proposed [2]. It will be interesting to compare its results with those for the staggered quarks.

The biggest new challenge for simulations at finite density is the sign problem. This is not an artifact of the lattice formulation, but is inherent in the continuum theory. Taking $N_{f}$ flavours of quarks, and denoting their chemical potentials by $\mu_{f}$, the partition function for QCD can be written as

$$
Z=\int D U \exp \left(-S_{G}\right) \prod_{f} \operatorname{Det} M\left(m_{f}, \mu_{f}\right)
$$

after integrating over the quarks fields. The thermal expectation value of an observable $\mathscr{O}$ is

$$
\langle\mathscr{O}\rangle=\frac{1}{Z} \int D U \exp \left(-S_{G}\right) \prod_{f} \operatorname{Det} M\left(m_{f}, \mu_{f}\right) \mathscr{O} .
$$

The usual methods of numerical simulations require $\operatorname{Det} M$ to be real and positive for every $U$. Generally, however, Det $M$ is a complex number for $\mu_{f} \neq 0$, although special lines can be found in the space of these parameters where the product of determinants is real. This presents a major block in extending the usual lattice techniques to the entire $T-\mu_{B}$ plane. Several approaches have been proposed in the past decade to deal with it; two parameter re-weighting [3], imaginary chemical potential [4], Taylor expansion [5], canonical ensemble method [6], and complex Langevin approach [7]. We employ the Taylor expansion approach [5] to obtain the results discussed here.

We have earlier presented results in full QCD with two flavours of staggered fermions of mass $m / T_{c}=0.1$ on $N_{t} \times N_{s}^{3}$ lattices, with $N_{t}=4$ and $N_{s}=8,10,12,16,24$ [8] and with a finer lattice spacing using $N_{t}=6$ with $N_{s}=12,18,24$ [9]. We have checked the earlier results of the MILC collaboration [10], which shows that our simulation parameters correspond to $m_{\pi} / m_{\rho}=$ $0.31 \pm 0.01$, implying a Goldstone pion mass of $230 \mathrm{MeV}$. Here we present results on an even finer lattice, $8 \times 32^{3}$, keeping mixed the ratio $m / T_{c}=0.1$ fixed. As before, we used the peak of the Polyakov loop susceptibility to define the reference coupling $\beta_{c}$. Our determination of $\beta_{c}$ is in 

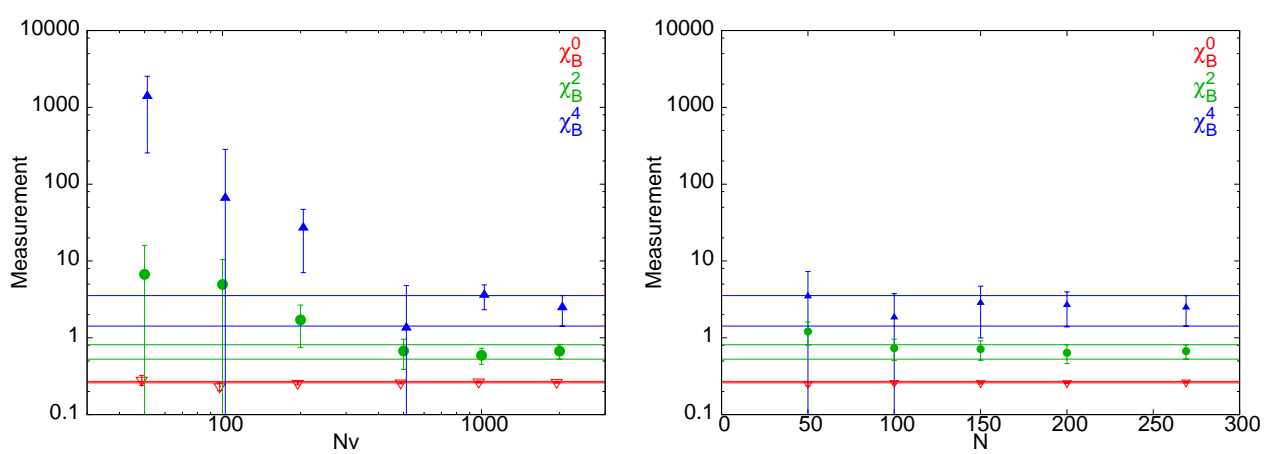

Figure 1: Coefficients of the Taylor expansion of the baryon number susceptibility, up to the 6th order nonlinear susceptibility, at fixed $\beta$ as the number of source vectors $N_{v}$ and the number of configurations $N$ are varied. At least 500 vectors are needed to get reasonable estimates of the non-linear susceptibilities.

agreement with previous results [11], and has higher precision. Plaquette values were measured at zero temperature at couplings corresponding to our finite temperature runs. These were used to set the relative lattice spacing and thereby define the temperature scale. Our simulations covered the temperature range $0.90 \leq T / T_{c} \leq 2.01$. Typically 100-200 independent configurations, separated by over 100 autocorrelation lengths were used to make measurements of physical quantities. This is an update of very preliminary results which were presented one year ago [12].

The QCD pressure $P$ has the following Taylor expansion

$$
\frac{P(\mu, T)}{T^{4}}-\frac{P(0, T)}{T^{4}}=\sum_{n_{u}, n_{d}} \chi_{n_{u}, n_{d}}(T) \frac{1}{n_{u} !}\left(\frac{\mu_{u}}{T}\right)^{n_{u}} \frac{1}{n_{d} !}\left(\frac{\mu_{d}}{T}\right)^{n_{d}}
$$

where the indices $n_{u}$ and $n_{d}$ denote the number of derivatives of the partition function with respect to the corresponding chemical potentials. The quantities $\chi_{n_{u}, n_{d}}$ are generalized (non-linear) susceptibilities. These are evaluated at $\mu_{f}=0 . \chi_{10}$ and $\chi_{01}$ are up and down quark number densities. $\chi_{20}$, $\chi_{02}$ and $\chi_{11}$ are called quark number susceptibilities. We can set $\mu_{u}=\mu_{d}=\mu_{B} / 3$ and $m_{u}=m_{d}$ in the expressions and so obtain a series for baryonic susceptibility from this expansion [8]. Its radius of convergence is what we look for.

\section{Controlling errors}

The statistically hard part of the computations is to evaluate the trace of products of fermion loops. It was pointed out earlier that the distribution of these quantities is strongly non-Gaussian, even if the distribution of the trace of a single loop is taken to be Gaussian. Errors in the measurements are controlled by two means: increasing the number of source vectors, $N_{v}$, used to evaluate the fermion trace in each configuration, and the number of configurations, $N$. It turns out that control over susceptibilities up to the 8th order requires us to use at least $N_{v}=1000$. Unless $N_{v}$ is sufficiently large, increasing $N$ is without commensurate value. Once errors in the $\chi_{n}$ 's are under control, estimates of the radius of convergence also come under control.

However, continuation of physical variables into the region near the critical point remain fraught with difficulties. Assume, for example, that we need an estimate of some quantity which 
has critical behaviour: $f(z)=\alpha\left(z-z_{c}\right)^{-\gamma}$. Assume also that there is a measurement of $z_{c}$ with a small error $\Delta z_{c}$. If the error in evaluating $f(z)$ is $\Delta f(z)$, then

$$
\frac{\Delta f(z)}{f(z)}=\frac{\gamma \Delta z_{c}}{z-z_{c}}
$$

So the error grows without bound as $z \rightarrow z_{c}$, i.e., in extrapolating towards the critical point. In order to obtain results with a fixed accuracy near the critical point, the statistics required grows as $1 /\left(z-z_{c}\right)^{2}$. This is the emergence of critical slowing down in Taylor expansion methods. This argument can be put on a theoretically sound footing.

\section{Lattice Results}
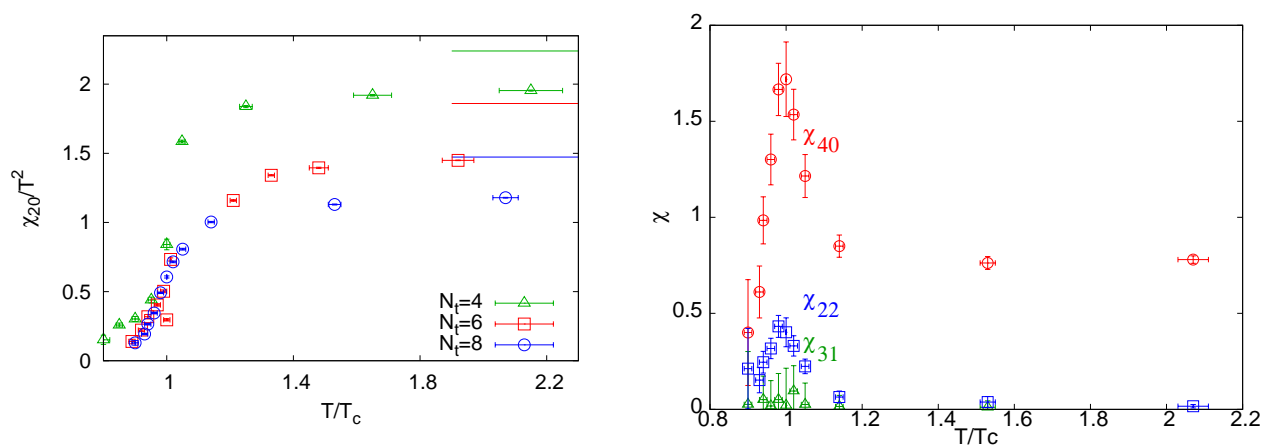

Figure 2: Comparison of baryon number susceptibility on $N_{t}=8$ with our earlier results on $N_{t}=6$ and 4 (Left panel). Various 4th order susceptibilities on the $N_{t}=8$ lattice (Right).

The panel on the left in Fig. 2 gives a comparison of our new results for the $N_{t}=8$ lattice for baryon number susceptibility with those for $N_{t}=6$ and 4 . At high temperature we also show lines to indicate the ideal gas values with these cutoffs. A more detailed comparison with weak coupling theory is given in the next section. The encouraging agreement between $N_{t}=8$ and 6 in the lowtemperature region, especially in the region where the critical point was located on the smaller of the two lattices, suggests that the dimensionless ratios which we employ in all our critical point determinations could possess a mild dependence on the lattice cutoff. The panel on the right shows the various fourth order susceptibilities we determined for the $N_{t}=8$ case. The broad features are essentially the same as observed on the coarser $N_{t}=6$ and 4 lattices.

Successive estimates for the radius of convergence are

$$
r_{n m}=\left(\frac{T^{n-2} \chi_{B}^{n} / n !}{T^{m-2} \chi_{B}^{m} / m !}\right)^{1 /(m-n)}
$$

We used terms up to 8th order in $\mu$ for doing so. A key point to note is that all coefficients of the series must be positive for the critical point to be at real $\mu$, and thus physical. If this condition is statisfied then a reasonable estimate of the critical point, $z_{c}=\mu / T$, can be obtained only when all the estimators are consistent with each other. The detailed expressions for all the terms, and the methods we use to evaluate them, are available in [8]. Our earlier determination of the critical 
point on $N_{t}=6$ resulted from the constancy for both the ratios defined above at $T / T_{c}=0.94$, with all the susceptibilities being positive, leading [9] to the coordinates of the endpoint (E) -the critical point-to be $T^{E} / T_{c}=0.94 \pm 0.01$, and $\mu_{B}^{E} / T^{E}=1.8 \pm 0.1$ for that lattice.
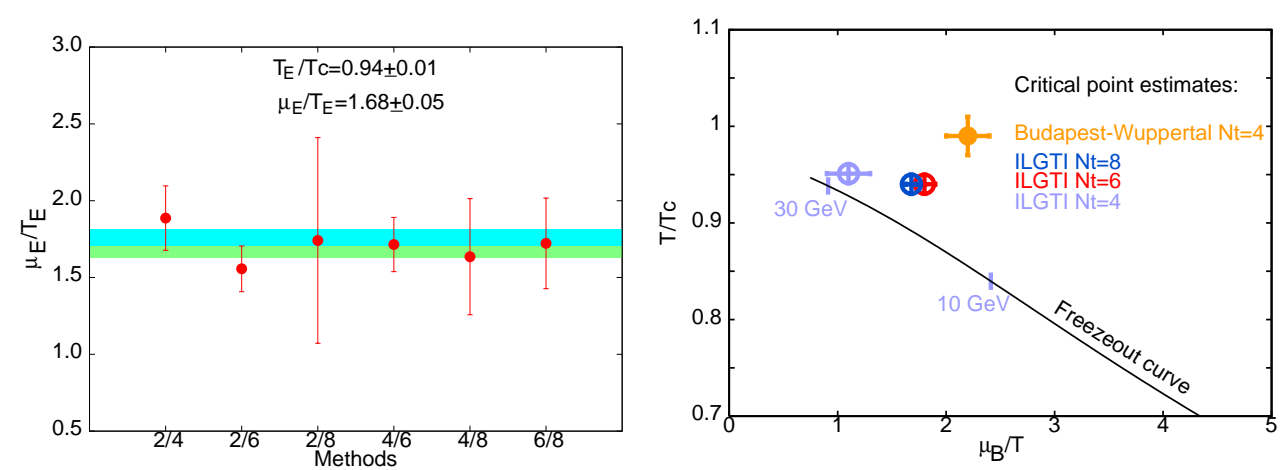

Figure 3: The radius of convergence estimates for $N_{t}=8$ (data) along with our final results for the $N_{t}=8$ (green band) and 6 critical chemical potential (cyan band) at the critical temperature of $T_{E} / T_{c}=0.94$ (Left panel). QCD phase diagram with all known lattice determinations for critical point (Right).

The panel in the left part of Fig. 3 displays our current estimates for the $N_{t}=8$, obtained by using the method described above. The two solid bands indicate our critical chemical potential estimates on $N_{t}=8$ (lower band) and $N_{t}=6$ (upper). Within the uncertainties of our measurement, the critical value of the temperature remains the same on the two lattices. On the finer lattice we see a minor shift to smaller $\mu_{B}$, albeit within $1-\sigma$, for the finer lattice. The panel on the right of the figure collects together the new results, old results for $N_{t}=6$ [9] and 4 [8], and the estimate of the critical point from the Budapest-Wuppertal collaboration [3], which used $N_{t}=4$.

\section{Susceptibilities at high temperature}

The susceptibilities are physically interesting observables, as they are connected to correlations and cumulants of quark number fluctuations $[8,13]$. In this section we show some susceptibilities of second and fourth order at $\mu_{B}=0$, that go into the estimation of the critical point. The left panel of Figure 4 shows the second order susceptibility $\chi_{20}$ in the high temperature side of the plasma, for temperatures $>1.1 T_{c}$. $\chi_{20}$ is proportional to baryon number fluctuation at $\mu_{B}=0$. Here, unlike in Fig. 2, we have normalized the data by the free theory result, $\chi_{20}^{\text {SB,Lat }} / T^{2}=1.473$, evaluated on $N_{t}=8$ lattice. We expect that normalizing this way might eliminate a large part of the cutoff effect at high temperature. In the same plot we also show results from finite temperature resummed perturbation theory calculations for the same quantity in various resummation schemes: hard thermal loop (HTL) scheme in leading (LO, Ref. [14]) and 3-loop order (NNLO, Ref. [15]), as well as a calculation based on dimensionally reduced effective theory (DR, Ref. [14]). At our higher temperatures, $\sim 2 T_{c}$, the nonperturbative results are still not in agreement with the perturbation theory results. While this could partly be due to cutoff effects in the lattice calculations, the spread between the calculations in different resummation schemes (and different orders) indicate that perturbation theory is not very reliable at these temperatures.

In the right panel of the figure, we show the off-diagonal susceptibility $\chi_{11}$ in units of $T^{2}$. This quantity is a marker of the correlation between $u$ and $d$ quantum numbers in the medium. As 

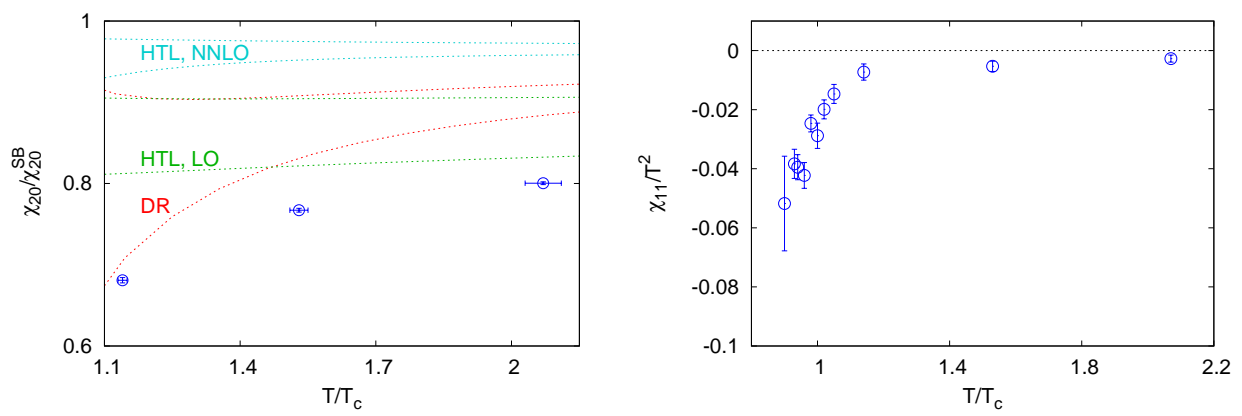

Figure 4: (Left) The second order susceptibility $\chi_{20}$ for $N_{f}=2$. The results are shown normalized by the lattice free theory result $\chi_{20}^{\text {SB,Lat }}$. Also shown are results from various resummed perturbation theory calculations. (Right) The off-diagonal susceptibility $\chi_{u d}$, plotted as function of $T^{2}$.
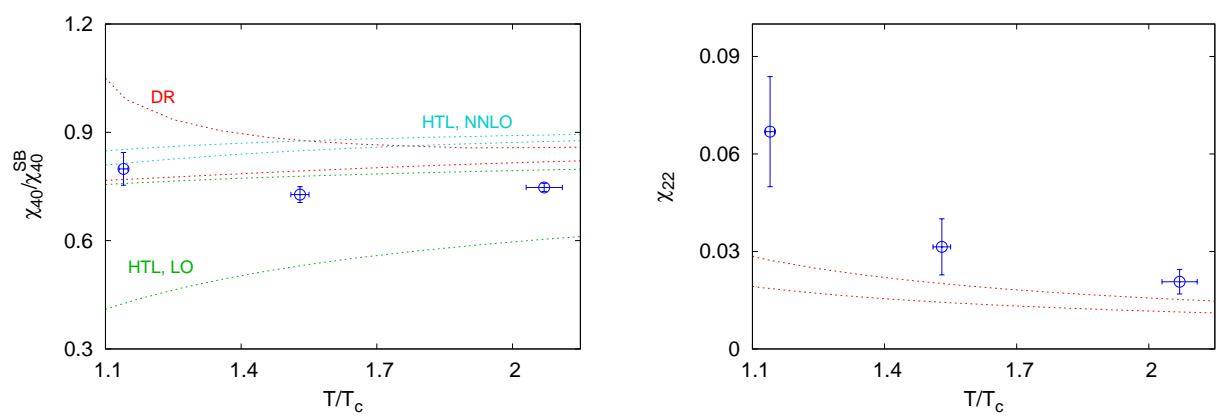

Figure 5: (Left) The fourth order nonlinear susceptibility $\chi_{40}$ for $N_{f}=2$, normalized by $\chi_{40}^{\text {SB,Lat }}$; also shown are perturbation theory calculations. (Right) The off-diagonal nonlinear susceptibility $\chi_{22}$, together with perturbation theory calculation.

seen in the figure, $\chi_{11}$ is negative in the confined phase, but quickly approaches zero in the plasma. In perturbation theory $\chi_{11} / T^{2} \sim g^{6} \log g$ [16], so its smallness in the high temperature side is in qualitative agreement with perturbation theory.

In Fig. 5 we show the behaviour of various nonlinear susceptibilities of fourth order in the high temperature phase of the plasma. In the left panel we show $\chi_{40}$, normalized by the free theory result for $N_{t}=8$ lattice, $\chi_{40}^{\mathrm{SB}, \mathrm{Lat}}=1.035$. The nonperturbative results are seen to be within the uncertainty band of the leading order HTL result at $2 T_{c}$, but slightly below the higher order dimensionally reduced calculation. In the right panel the off-diagonal quantity $\chi_{22} / T^{2}$ is shown, together with the results of Ref. [14] from a calculation based on dimensional reduction. Near 2 $T_{c}$ the nonperturbative results are seen to be close to, but just outside, the uncertainty band of the perturbation theory calculation.

\section{Summary}

We have presented lattice results for the non-linear quark and baryon number susceptibilities in two-flavour QCD with light pions with $N_{t}=8$. We find indications from the Taylor series expansion for the existance of a critical point. Our results for the location of this point is consistent with that 
obtained earlier using $N_{t}=6$. This suggests that the continuum limit may be reached with little change, and the current estimates of the critical point could be robust.

This work was done on the Blue Gene P of Indian Lattice Gauge Theory Initiative, Tata Institute (TIFR), Mumbai. We gratefully acknowledge financial and technical support of TIFR.

\section{References}

[1] M. Asakawa and K. Yazaki, Nucl. Phys. A504 (1989) 668.

[2] R. V. Gavai and S. Sharma, Phys. Lett. B716 (2012) 446.

[3] Z. Fodor \& S. Katz, JHEP 0203 (2002) 014, and JHEP 0404 (2004) 050.

[4] Ph. de Forcrand \& O. Philipsen, Nucl. Phys. B642 (2002) 290; M. -P. Lombardo \& M. D’Elia, Phys. Rev. PR D67 (2003) 014505.

[5] R. V. Gavai and S. Gupta, Phys. Rev. D68 (2003) 034506; C. Allton et al.,Phys. Rev. D68 (2003) 014507.

[6] K. -F. Liu, Int. J. Mod. Phys. B16 (2002) 2017; S. Kratochvila and Ph. de Forcrand, PoS LAT2005 (2006) 167.

[7] G. Aarts, E. Seiler and I. O. Stamatescu, Phys. Rev. D 81 (2010) 054508 and references therein.

[8] R. V. Gavai and S. Gupta, Phys. Rev. D71 (2005) 114014.

[9] R. V. Gavai and S. Gupta, Phys. Rev. D78 (2008) 114503.

[10] S. Gottlieb et al., Phys. Rev. Lett. 59 (1987) 1513; Phys. Rev. D 38 (1988) 2245.

[11] S. Gottlieb et al., Phys. Rev. D47 (1993) 3619.

[12] S. Datta, R. V. Gavai and S. Gupta, Nucl. Phys. A904-905 (2013) 883c.

[13] S. Gupta, PoS, CPOD2009 (2009) 025; R. V. Gavai and S. Gupta, Phys. Lett. B696 (2011) 459.

[14] S. Mogliacci, J. O. Anderson, M. Strickland, N. Su and A. Vuorinen, arXiv:1307.8098.

[15] N. Haque, J. O. anderson, M. G. Mustafa, M. Strickland and N. Su, arXiv:1309.3968.

[16] J.-P. Blaizot, E. Iancu and A. Rebhan, Phys. Lett. B523 (2001) 143. 